\title{
Black holes that repel
}

\author{
H. Lü, ${ }^{1, *}$ Zhao-Long Wang, ${ }^{2,3, \dagger}$ and Qing-Qing Zhao ${ }^{1, \$}$ \\ ${ }^{1}$ Center for Joint Quantum Studies, School of Science, Tianjin University, Tianjin 300350, China \\ ${ }^{2}$ Institute of Modern Physics, Northwest University, XiAn 710069, China \\ ${ }^{3}$ Shaanxi Key Laboratory for Theoretical Physics Frontiers, XiAn 710069, China
}

(Received 22 January 2019; published 29 May 2019)

\begin{abstract}
The recent observation that black holes in certain Einstein-Maxwell-dilaton (EMD) theories can violate the entropy super-additivity led to the suggestion that these black holes might repel each other. In this paper, we consider EMD theories with two Maxwell fields $A_{i}$, with general exponential couplings $\exp \left(a_{i} \phi\right)$ in their kinetic terms. We calculate the gravielectrostatic force between charged black holes $\left(m_{1}, e_{1}\right)$ and $\left(M_{2}, Q_{2}\right)$; the former is sufficiently small and can be treated as a pointlike object. We find there is a potential barrier caused by the dilaton coupling at $r_{0}$ outside the black hole horizon $r_{+}$, provided that $-a_{1} a_{2}>2(D-3) /(D-2)$. As the black hole approaches extremality, both $r_{+}$and $r_{0}$ vanish, the barrier becomes infinitesimally thin but infinitely high, and the two black holes repel each other in the whole space. There is no electrostatic force between them; the dilaton is the antigravity agent. Furthermore we find that the exact constraint on $a_{1} a_{2}$ can be derived from the requirements that two-charged extremal black holes have a fission bomb like mass formula and the violation of entropy super-additivity can occur. The two very different approaches give a consistent picture of the black hole repulsion.
\end{abstract}

DOI: 10.1103/PhysRevD.99.101502

\section{INTRODUCTION}

Newton's law of universal gravitation states that any two massive objects attract by gravity. This remains largely true in Einstein's theory of general relativity (GR) but with some subtleties. The centrifugal force is viewed as fictitious in Newton's theory, it is an intrinsic effect of spacetime structure in GR. It is thus advantageous to simplify the discussion on the effective force between two objects by restricting the motion with no relative angular momentum. Furthermore, in de Sitter spacetimes or any universe with accelerating expansion, two black holes in sufficient separation will move away from each other by the negative pressure. In order to avoid this effect, we shall focus only on the black holes that are asymptotic to the Minkowski spacetime.

In Newtonian gravity, the attractive force can be perfectly balanced by the Coulomb's electrostatic force, the other long-range force in nature. In particular, a set of charged particles of mass $m$ and charge $e$ can all be in static balance when $m=e$. This property remains in GR even

\footnotetext{
*mrhonglu@gmail.com

†zlwang@nwu.edu.cn

zhaoqq@tju.edu.cn
}

Published by the American Physical Society under the terms of the Creative Commons Attribution 4.0 International license. Further distribution of this work must maintain attribution to the author(s) and the published article's title, journal citation, and DOI. Funded by SCOAP. when the theory becomes highly nonlinear. The $m=e$ particles become the extremal Reissner-Nordström (RN) black holes. These black holes continue to experience no force and statically they can be arbitrarily located in space. The $m>e$ particles become nonextremal black holes with Hawking temperature. The $m<e$ particles, such as an electron, remain particlelike with naked curvature singularity.

There is no doubt that two Schwarzschild black holes always attract; in fact, they can remain static only with a naked strut singularity $[1,2]$. The situation becomes more complicated for RN black holes. A direct calculation of the force is formidable. When one of the two black holes is sufficiently small, it can be treated as a pointlike object. It was shown that there can be no static equilibrium for a charged particle with $m>e$ outside the event horizon of an $\mathrm{RN}$ black hole [3]. This is indicative that two RN black holes always attract. (Hairy magnetic black holes were constructed to lower the mass so that two such black holes can be repulsive at large separation, see, e.g. [4-6].) The attractiveness is also supported by the fact that the RN black hole entropy is a super-additive function of its mass and charge. A black hole's entropy is in general a function of its conserved quantities $Q^{i}$, which include the mass, charge and angular momentum. If we imagine that two black holes with $Q_{1}^{i}$ and $Q_{2}^{i}$ join to become a bigger black hole, entropy super-additivity states $S\left(Q_{1}^{i}+Q_{2}^{i}\right) \geq$ $S\left(Q_{1}^{i}\right)+S\left(Q_{2}^{i}\right)$ [7]. This is related to Hawking's black hole area law and it is consistent with the assumption that black holes are mutually attractive and merging together is a 
physical process that increases the entropy. Indeed $\mathrm{RN}$ black holes, like the Schwarzschild, satisfy the entropy super-additivity.

However, it turns out that the super-additivity rule can be violated [7] by the Kaluza-Klein dyonic black holes in four dimensions [8]. Furthermore, the mass of the extremal dyon is larger than the sum of the masses of the individual extremal electric and magnetic ones. The dyon is thus a bound state of electric and magnetic black holes with negative binding energy, analogous to a fission bomb $[9,10]$. The issue of generating angular momentum by the separation of the electric and magnetic charges can be circumvented if we consider two Maxwell fields, with both carrying electric charges. Further black hole fission bombs in Einstein-Maxwell-dilaton (EMD) theories that violate the entropy super-additivity were constructed in [11]. These results led one to propose that two black holes might not always attract [12], and the dilaton may play the role of antigravity $[9,13]$.

In this paper, we consider EMD theories with two Maxwell fields $A_{1}$ and $A_{2}$, both of which couple to the dilation $\phi$ nonminimally, with exponential couplings in their kinetic terms. We calculate the force on a charged particle coupled to $A_{1}$ by the black hole carrying only the $A_{2}$ charge, and hence there is electrostatic force between them. We find that there is an unstable equilibrium when the black hole becomes extremal or sufficiently near extremum, indicating repulsive force exists between the black hole and the charged particle. Furthermore, the particle satisfies the black hole mass-charge bound and hence two such black holes do repel each other, with antigravity mediated by the dilaton.

In Sec. II, we present the formalism for calculating the gravielectrostatic force. In Secs. III and IV, we study black hole interactions in EMD theories with one and two Maxwell fields respectively. We conclude the paper in Sec. V.

\section{GRA VIELECTROSTATIC FORCE}

We set up here the formalism for calculating the gravielectrostatic force between a charged black hole and a point particle outside the horizon. (See e.g. [14].) We shall consider only the static and spherically symmetric black holes. The most general ansatz in some appropriate metric frame takes the form

$d s_{D}^{2}=-h(r) d t^{2}+\frac{d r^{2}}{f(r)}+\rho(r)^{2} d \Omega_{\text {sphere }}^{2}, \quad A=\psi(r) d t, \ldots$,

where $\psi(r)$ is the electrostatic potential, and the ellipses denote other matter fields that are not relevant for our purpose. The motion of a particle of mass $m$ couple to $A$ with charge $e$ is governed by the action

$$
S=-m \int d \tau\left(\sqrt{-g_{\mu \nu} \frac{d x^{\mu}}{d \tau} \frac{d x^{\nu}}{d \tau}}+\frac{e}{m} A_{\mu} \frac{d x^{\mu}}{d \tau}\right) .
$$

We are interested in static equilibria; therefore, we focus on the radial motion only. The relevant action can be written as

$$
S=m \int d t L, \quad L=-\sqrt{h(r)-\frac{\dot{r}^{2}}{f(r)}}-\frac{e}{m} \psi(r),
$$

where the radial variable $r$ is now a function of the asymptotic physical time $t$ and a dot is a derivative with respect to $t$. To derive the gravielectrostatic force, we restrict our attention to $\dot{r}^{2} \ll 1$, in which case, the effective Lagrangian is

$L=\frac{\dot{r}^{2}}{2 \sqrt{h(r)} f(r)}-V_{\mathrm{eff}}(r), \quad V_{\mathrm{eff}}=\sqrt{h(r)}+\frac{e}{m} \psi(r)$.

This is a Newtonian system with an $r$-dependent mass and potential $V_{\text {eff }}$. When the charge and mass satisfy the ratio at certain $r_{0}$, namely

$$
\frac{e}{m}=-\left.\frac{(\sqrt{h})^{\prime}}{\psi^{\prime}}\right|_{r=r_{0}},
$$

it is a static equilibrium. The existence of such an equilibrium implies that the particle must experience an overall repulsive force somewhere in its vicinity. We must require $r_{0}$ be outside the horizon, and furthermore, the particle mass be sufficiently larger than its charge so that it is a small black hole rather than an electronlike particle. For the linear radial perturbation $r(t)=r_{0}+\epsilon(t)$, the solution is $\epsilon \sim \exp ( \pm \lambda t)$, with

$$
\lambda^{2}=\left.\sqrt{h} f\left(\frac{\psi^{\prime \prime}}{\psi^{\prime}}(\sqrt{h})^{\prime}-(\sqrt{h})^{\prime \prime}\right)\right|_{r=r_{0}} .
$$

The characteristics of the equilibrium is determined by the sign of $\lambda^{2}$.

It is worth pointing out that in string-inspired EMD theories, there can be multiple Maxwell fields. If the particle of $(m, e)$ does not couple to $A$, the second term in the action (2.2) drops out and there is no electrostatic force. We then have

$$
h^{\prime}\left(r_{0}\right)=0, \quad \lambda^{2}=-\frac{1}{2} f\left(r_{0}\right) h^{\prime \prime}\left(r_{0}\right) .
$$

\section{EMD THEORY}

We first consider the EMD theory in general $D$ dimensions: 


$$
\mathcal{L}=\sqrt{-g}\left(R-\frac{1}{2}(\partial \phi)^{2}-\frac{1}{4} e^{a \phi} F^{2}\right), \quad F=d A
$$

Introducing $N$ by $a^{2}=\frac{4}{N}-\frac{2(D-3)}{D-2}$, charged black holes can be written as

$$
\begin{aligned}
d s^{2} & =-H^{-\frac{D-3}{D-2} N} \tilde{f} d t^{2}+H H^{\frac{N}{D-2}}\left(\frac{d r^{2}}{\tilde{f}}+r^{2} d \Omega^{2}\right), \\
A & =\psi d t, \quad \phi=\frac{1}{2} N a \log H, \\
\tilde{f} & =1-\frac{\mu}{r^{D-3}}, \quad \psi=\frac{\sqrt{N q(\mu+q)}}{r^{D-3} H}, \quad H=1+\frac{q}{r^{D-3}} .
\end{aligned}
$$

Note that for $a \neq 0$, we have $\rho \rightarrow 0$ as $r \rightarrow 0$; therefore, $r$ can be qualitatively treated as the black hole radius. The horizon is at $r_{+}=\mu^{1 /(D-3)}$, with surface gravity
$\kappa=(D-3) /\left(2 r_{+} H_{+}^{N / 2}\right)$, where $H_{+}=H\left(r_{+}\right)$. The black hole thermodynamical quantities are

$$
\begin{aligned}
T & =\frac{\kappa}{2 \pi}, \quad S=\frac{1}{4} \Omega_{D-2} H_{+}^{\frac{1}{2} N} r_{+}^{D-2}, \quad \Psi=\sqrt{\frac{N q}{q+r_{+}^{D-3}}}, \\
M & =\frac{\Omega}{16 \pi}\left((D-2) r_{+}^{D-3}+(D-3) N q\right), \\
Q & =\frac{(D-3) \Omega}{16 \pi} \sqrt{N q\left(q+r_{+}^{D-3}\right)} .
\end{aligned}
$$

They satisfy the first law $d M=T d S+\Psi d Q$. Here $\Omega$ denotes the volume of the unit $S^{D-2}$. The solution is extremal when $r_{+}=0$, corresponding to $M_{\text {ext }}=\sqrt{N} Q$. In this limit, $H$ can be any harmonic function of the Euclidean transverse space.

For general nonextremal black holes, we have $M>M_{\text {ext }}$. [A particle with $(m, e)$ is a small black hole if $m \geq \sqrt{N} e$.] The entropy depends on the mass and charge, namely

$$
\begin{aligned}
S & =\frac{1}{4} \Omega r_{+}^{D-2}\left(1-\frac{D-2}{(D-3) N}+\frac{16 \pi M}{(D-3) \Omega N r_{+}^{D-3}}\right)^{N / 2}, \\
r_{+}^{D-3} & =\frac{8 \pi\left[((D-3) N-2(D-2)) M+\sqrt{(D-3)^{2} N^{2} M^{2}-4(D-2) N((D-3) N-D+2) Q^{2}}\right]}{(D-2)((D-3) N-D+2) \Omega} .
\end{aligned}
$$

Although we have not proven analytically the entropy super-additivity for the general case, we have not seen any counterexamples in a thorough numerical analysis. Note that when we split $(M, Q)=\left(M_{1}+M_{2}, Q_{1}+Q_{2}\right)$, the mass-charge bound should be held not only for $(M, Q)$ but also for $\left(M_{1}, Q_{1}\right)$ and $\left(M_{2}, Q_{2}\right)$.

We now turn to calculate the gravielectrostatic force on a charged particle outside the black hole. Naively, one might simply substitute the metric (3.2) into the formalism of Sec. II. However, there is a subtlety in the EMD theory. There will be an additional $e^{\alpha \phi}$ type of dilation coupling in the particle world line action. The value of $\alpha$ can be determined by noticing that there is a metric frame associated with the charged particle itself. In its particle frame, the corresponding electric charge can be simply expressed as the usual EM theory $Q=\int * F$ without any further $\phi$ dependence. Therefore, the particle frame metric $\hat{g}_{\mu \nu}$ just given by the conformal transformation from the Einstein frame $g_{\mu \nu}=e^{a \phi} \hat{g}_{\mu \nu}$. In its own frame, the charged particle is minimally coupled to the background gravity as well as the gauge field as in (2.2). This is analogous to the string action that should be written in the string frame. (See, e.g. [15].) In the particle frame, the Lagrangian becomes

$$
\begin{aligned}
\hat{\mathcal{L}}= & \sqrt{-\hat{g}} e^{\frac{1}{2} 12(D-2) a \phi}\left(\hat{R}-\frac{1}{4} F^{2}\right. \\
& \left.-\frac{1}{2}\left[1-\frac{1}{2}(D-1)(D-2) a^{2}\right](\partial \phi)^{2}\right),
\end{aligned}
$$

and the metric functions of the black hole become

$$
\hat{h}=\frac{\tilde{f}}{H^{2}}, \quad \hat{f}=H^{2-N} \tilde{f}, \quad \hat{\rho}^{2}=r^{2} H^{N-2} .
$$

A further important property is that the hatted particle action preserves the symmetry $\phi \rightarrow \phi+c$ and $A \rightarrow e^{-\frac{1}{2} a c} A$ of the original Lagrangian. Following Sec. II, we find the equilibrium $r_{0}$ is determined by

$$
\frac{e}{m}=\frac{2 q r_{0}^{D-3}+\left(r_{0}^{D-3}-q\right) r_{+}^{D-3}}{2 \sqrt{N q r_{0}^{D-3}\left(r_{0}^{D-3}-r_{+}^{D-3}\right)\left(r_{+}^{D-3}+q\right)}}
$$

In the extremal $r_{+}=0$ limit, we have the extremal $e / m=$ $1 / \sqrt{N}$, which is independent of $r_{0}$. This is the manifestation of no force between two extremal black holes. For general nonextremal black holes with $r_{+}>0$, we require that $r_{0} \geq r_{+}$. The charge/mass ratio is a monotonically decreasing function of $r_{0} \in\left(r_{+}, \infty\right)$. Thus we have

$$
\frac{e}{m} \geq \frac{2 q+r_{+}^{D-3}}{2 \sqrt{N q\left(q+r_{+}^{D-3}\right)}} \geq \frac{1}{\sqrt{N}} .
$$

In other words, the particle that has a static equilibrium cannot be a black hole. Two nonextremal black holes carrying the same type of charges always attract, consistent with our earlier observation that they satisfy the entropy 
super-additivity. The $a=0$ case reproduces the conclusion for the RN black hole [3]. It is worth seeing that equilibria of the particles are all unstable with

$$
\lambda=\left(\frac{r_{+}}{r_{0}}\right)^{D-2}\left(\frac{H\left(r_{+}\right)}{H\left(r_{0}\right)}\right)^{N / 2} \kappa,
$$

satisfying the bound $\lambda<\kappa$, proposed in [14].

\section{EMD THEORY WITH TWO MAXWELL FIELDS}

We now generalize Sec. III by introducing another Maxwell field. The Lagrangian in the Einstein frame is

$$
\begin{aligned}
\mathcal{L} & =\sqrt{-g}\left(R-\frac{1}{2} e^{a_{1} \phi} F_{1}^{2}-\frac{1}{2} e^{a_{2} \phi} F_{2}^{2}\right), \\
F_{i} & =d A_{i}, \quad a_{i}^{2}=\frac{4}{N_{i}}-\frac{2(D-3)}{D-2} .
\end{aligned}
$$

For later purposes, we introduce a parameter $\zeta$, defined by

$$
\zeta=-\frac{1}{2} a_{1} a_{2}-\frac{D-3}{D-2} .
$$

Singly charged black holes associated with either $A_{1}$ or $A_{2}$ are given in (3.2) with the fields and constants labeled by subscripts "1" or "2" appropriately. When $\zeta=0$, exact solutions can be constructed carrying both charges, and the no-force condition exists in the extremal limit (see, e.g. [16]). For $\zeta \neq 0$, general analytical solutions are hard to come by. In $D=4$ and $a_{2}=-a_{1}$, approximate solutions were constructed in [11]. When $a_{1} a_{2}$ is negative, an exact solution always exists for appropriately fixed charges. To be specific, when

$$
Q_{i}=\sqrt{-\frac{\epsilon_{i j} a_{j}}{a_{1}-a_{2}}} Q,
$$

the solution becomes the RN black hole with charge $Q$. (Here we take $a_{1}$ and hence $-a_{2}$ to be positive.) The twocharge extremal solution is a bound state of two extremal $\left(Q_{1}, 0\right)$ and $\left(0, Q_{2}\right)$ black holes, with the binding energy

$\Delta M=M_{1}+M_{2}-M=\sqrt{N_{1}} Q_{1}+\sqrt{N_{2}} Q_{2}-\sqrt{\frac{2(D-2)}{D-3}} Q$.

We find that $\Delta M(>,=,<) 0$ corresponds to $\zeta(<,=,>) 0$ respectively. In string theories, where $\zeta=0$, vanishing $\Delta M$ is a consequence of the no-force condition between the supersymmetric intersecting $p$-branes. The $\Delta M<0$ and $\Delta M>0$ cases were referred to as fission and fission bombs in [10]. The $\zeta>0$ case with negative binding energy suggests that the extremal black holes are repulsive.
In fact, in $D=4$, and $a_{1}=-a_{2}$, for which $\zeta=a_{1}^{2}-1$, it was demonstrated numerically that entropy superadditivity can be violated for $\zeta>0$ [11]. In particular, for $a_{1}=\sqrt{3}$, corresponding to the Kaluza-Klein theory, the violation can be established analytically [7].

We now calculate the static force between the black holes $\left(M_{1}, Q_{1}\right)$ and $\left(M_{2}, Q_{2}\right)$, associated with $A_{1}$ and $A_{2}$ respectively, and hence there is no electrostatic force between them. Assuming that $\left(M_{1}, Q_{1}\right)=\left(m_{1}, e_{1}\right)$ are sufficiently small to be a point object, we can apply (2.7). In the particle frame associated with $A_{1}$, the relevant metric functions of the black hole $\left(M_{2}, Q_{2}\right)$ are

$$
\hat{h}=H_{2}^{\zeta N_{2}} \tilde{f}, \quad \hat{f}=H_{2}^{-(\zeta+1) N_{2}} \tilde{f} .
$$

From the asymptotic behavior of $\hat{h}$, the antigravity becomes evident for $\zeta>0$. To be specific, in the $r_{+}=0$ extremal case, we have no force when $\zeta=0$, for which $h=1$. In general, we find that there is an equilibrium $\hat{h}^{\prime}\left(r_{0}\right)=0$, yielding

$$
\left(\frac{r_{0}}{r_{+}}\right)^{D-3}=1+\frac{q_{2}+r_{+}^{D-3}}{\zeta N_{2} q_{2}-r_{+}^{D-3}}
$$

Thus for $\zeta \leq 0$, the equilibrium is always inside the horizon, implying that the two objects always attract. When $\zeta>0$, on the other hand, the equilibrium can be outside the horizon for sufficiently small $r_{+}$. In particular, for $r_{+} \in\left(0,\left(\zeta N_{2} q_{2}\right)^{\frac{1}{D-3}}\right)$, we have $r_{0} \in(0, \infty)$. In terms of the black hole mass-charge relation, we have

$$
\sqrt{N_{2}} \leq \frac{M_{2}}{Q_{2}} \leq \frac{\sqrt{N_{2}}((D-3)+(D-2) \zeta)}{(D-3) \sqrt{1+N_{2} \zeta}}
$$

Note that as the black hole $\left(M_{2}, Q_{2}\right)$ approaches extremality $r_{+} \rightarrow 0$, the equilibrium $r_{0}$ also approaches zero with $r_{0} / r_{+} \sim 1+1 /\left(\zeta N_{2}\right)$. All the equilibria are unstable, with

$$
\lambda^{2}=\frac{(D-3)^{2} r_{+}^{2(D-3)}\left(\zeta N_{2} q_{2} r_{+}^{3-D}-1\right)^{\frac{2(D-2)}{D-3}}}{2 N_{2}^{N_{2}+1} q_{2}^{\frac{2(D-2)}{D-3}} \zeta^{N_{2}+1}\left(\zeta N_{2}+1\right)^{\frac{D-1}{D-3}-N_{2}}\left(q_{2} r_{+}^{3-D}+1\right)^{N_{2}}} .
$$

(Unlike the previous case, the $\lambda<\kappa$ bound can now be violated.) Thus for a charged particle $\left(m_{1}, e_{1}\right)$ outside the black hole $\left(M_{2}, Q_{2}\right)$, satisfying (4.7), there is a potential barrier of the gravielectrostatic force at certain $r_{0}>r_{+}$. As the black hole becomes extremal, both $r_{+}$and $r_{0}$ approach zero, and the barrier becomes infinitesimally thin but infinitely high. The two objects are then repulsive in the whole space. This result is independent of the mass and charge ratio of $\left(m_{1}, e_{1}\right)$, indicating that the repulsion can exist between two such black holes, mediated by the dilaton. 


\section{CONCLUSIONS}

We considered a class of EMD theories with two Maxwell fields. We calculated the static force between one black hole of mass and charge $\left(M_{2}, Q_{2}\right)$ and the other of $\left(m_{1}, e_{1}\right)$ that was sufficiently small and could be treated as pointlike. It is clear that for large enough mass/charge ratio, black holes will always attract. However, in the extremal or near extremal cases, the sign of the force depends on the $\zeta$ parameter (4.2) associated with the dilaton coupling constants $\left(a_{1}, a_{2}\right)$. For $\zeta<0$, the black holes always attract. This includes the case when two black holes carrying the same type of charges $\left(a_{1}=a_{2}\right)$, as demonstrated in Sec. III. For $\zeta=0$, two black holes in general attract, except when both are extremal, in which case, there is no force between them. This includes black holes that are supersymmetric intersections of $p$-branes. Repulsion occurs when $\zeta>0$ and the black holes are extremal or sufficiently nearly extremal. This reproduces precisely the same result from examining the black hole mass and entropy formulas: the two-charge extremal or near-extremal black holes with $\zeta>0$ are like bound states with negative binding energy and furthermore they can violate the entropy super-additivity. The two totally different approaches point to the same picture that these black holes will naturally split, like fission bombs. When $a_{2}=-a_{1}$, the two electrically charged black holes can be viewed as dyonic black holes associated with a single field strength. We thus demonstrated concretely that repulsion exists between the dyonic black holes in the Kaluza-Klein theory, which can be embedded in supergavities. The Kaluza-Klein dyon is the only known exact solution that violates the entropy super-additivity. The lacking of such exact solutions makes it difficult to study systematically the relation between the violation of the super-additivity and the existence of the black hole repulsion; it is nevertheless an interesting subject to investigate.

It should be emphasized that the two black holes of $\left(M_{1}, Q_{1}\right)$ and $\left(M_{2}, Q_{2}\right)$ do not have electrostatic force between them, since they are charged under different Maxwell fields. The repulsion is mediated by the dilaton, and our result is indeed sensitive to the parameter $\zeta$. Our conclusion opens up a variety of related questions in black hole physics, such as black hole horizon area and the information paradox when a black hole may break up like a bomb. Since EMD theories are inspired by the strings where the black holes are lower-dimensional artifacts of branes, the issue of black hole repulsion and related topics can also be addressed by the brane dynamics in strings and/or M-theory. In fact, it is known that there are always dilaton forces between the D-branes in a $D p / D q$ system when $p \neq q$. (There is no electrostatic interaction between $D p$ and $D q$ branes when $p \neq q$.) For $D 0 / D 4$, the repulsive dilaton force cancels the gravity force and makes the corresponding bound state to be marginal. For $D 0 / D 6$ the repulsive dilaton force is larger than the gravity force and thus the $D 0 / D 6$ bound state is unstable, which is in fact a string origin of the Kaluza-Klein dyon repulsion mentioned earlier. More systematic analysis of dilaton mediated force in the brane dynamics will be presented in our incoming work [17].

\section{ACKNOWLEDGMENTS}

We are grateful to Gary Gibbons for discussions. H. L. and Q. Q.Z. are supported in part by NSFC Grants No. 11875200 and No. 11475024. Z. L.W. is supported in part by NSFC Grants No. 11305125 and No. 11447607 and Basic Research Program of Natural Science of Shaanxi Province Grants No. 2019JM-026.
[1] G. W. Gibbons, The motion of black holes, Commun. Math. Phys. 35, 13 (1974).

[2] H. Müller zum Hagen and H. J. Seifert, Two axisymmetric black holes cannot be in static equilibrium, Int. J. Theor. Phys. 8, 443 (1973).

[3] Q. Q. Zhao, Y.Z. Li, and H. Lü, Static equilibria of charged particles around charged black holes: Chaos bound and its violations, Phys. Rev. D 98, 124001 (2018).

[4] K. M. Lee, V. P. Nair, and E. J. Weinberg, A Classical Instability of Reissner-Nordstrom Solutions and the Fate of Magnetically Charged Black Holes, Phys. Rev. Lett. 68, 1100 (1992).

[5] J. H. Horne and G. T. Horowitz, Black holes coupled to a massive dilaton, Nucl. Phys. B399, 169 (1993).

[6] K. M. Lee and E. J. Weinberg, Nontopological Magnetic Monopoles and New Magnetically Charged Black Holes, Phys. Rev. Lett. 73, 1203 (1994).
[7] M. Cvetič, G. W. Gibbons, H. Lü, and C. N. Pope, Killing horizons: Negative temperatures and entropy super-additivity, Phys. Rev. D 98, 106015 (2018).

[8] G. W. Gibbons and D. L. Wiltshire, Black holes in KaluzaKlein theory, Ann. Phys. (N.Y.) 167, 201 (1986); Erratum, Ann. Phys. (N.Y.) 176, 393(E) (1987).

[9] G. W. Gibbons and R. E. Kallosh, Topology, entropy and Witten index of dilaton black holes, Phys. Rev. D 51, 2839 (1995).

[10] H. Lü and C. N. Pope, Fission and fusion bound states of $p$-brane solitons, arXiv:hep-th/9606047.

[11] W. J. Geng, B. Giant, H. Lü, and C. N. Pope, Mass of dyonic black holes and entropy super-additivity, arXiv: 1811.01981.

[12] H. Lü, Do two black holes always attract?, at Yangzhou University (2018), Huzhou Normal University (2018) and Chongqing University (2018). 
[13] G. W. Gibbons and C. G. Wells, Antigravity bounds and the Ricci tensor, arXiv:gr-qc/9310002.

[14] K. Hashimoto and N. Tanahashi, Universality in chaos of particle motion near black hole horizon, Phys. Rev. D 95, 024007 (2017).

[15] M. J. Duff, R. R. Khuri, and J. X. Lu, String solitons, Phys. Rep. 259, 213 (1995).
[16] H. Lü, Charged dilatonic AdS black holes and magnetic $\mathrm{AdS}_{D-2} \times R^{2}$ vacua, J. High Energy Phys. 09 (2013) 112.

[17] H. Lü and Z.L. Wang, Higher-dimensional origins of the Kaluza-Klein dyon repulsion (to be published). 